\title{
Molecular Cloning of a cDNA Coding for Feline Liver Xanthine Dehydrogenase
}

\author{
Shuichi TSUCHIDA ${ }^{1)}$, Rieko YAMADA ${ }^{1)}$, Shigenori IKEMOTO $^{1)}$ and Masahiro TAGAWA ${ }^{1)}$ \\ ${ }^{1)}$ Division of Veterinary Surgery, Nippon Veterinary and Animal Science University, 1-7-1 Kyonan-cho, Musashino, Tokyo 180-8602, \\ Japan
}

(Received 24 July 2000/Accepted 1 December 2000)

ABSTRACT. A cDNA coding for feline liver xanthine dehydrogenase(XDH, EC1.1.1.204) was amplified by RT-PCR and cloned for determining the sequence. The clones contained an open reading frame of 4002 base pairs encoding 1333 amino acid residues. The calculated molecular weight and isoelectric point were approximately $146 \mathrm{kDa}$ and 7.0. Comparison of the deduced amino acid sequences indicated remarkable high homology, i.e., the amino acid residues of feline XDH shared approximately $90 \%, 87 \%, 87 \%$ and $86 \%$ identity with those of human, bovine, rat and mouse, respectively. The anino acid sequences of two putative iron-sulfur centers, one NAD binding site and one molybdenum binding site were well conserved among mammalian animals.

KEY WORDS: cDNA, feline, xanthine dehydrogenase.

J. Vet. Med. Sci. 63(3): 353-355, 2001

Xanthine dehydrogenase (XDH, EC1.1.1.204) is an enzyme that has a primary biochemical role in purine catabolism. XDH catalyzes the last two steps of purine degradation to form xanthine from hypoxanthine and uric acid from xanthine. Mammalian XDH exists mainly as an NADdependent form in tissues. However, during the purification, most of the enzymes are converted into an $\mathrm{O}_{2}$-dependent form (xanthine oxidase: XO, EC1 2.2.2) by irreversible limited proteolysis or reversible oxidation $[1,6,11,16]$. $\mathrm{XDH}-\mathrm{XO}$ conversion occurs in various pathological condition in vivo, in particular, ischemia-reperfusion injury in various organs $[9,11]$. $\mathrm{XDH}$ is a homodimer of a subunit with molecular weight of approximately $150 \mathrm{kDa}$, which is a metalloflavoenzyme containing one molybdenum binding site, two iron-sulfur centers and one flavin adenine dinucleotide center (FAD) $[11,18]$. The cDNA sequences coding the full amino acid sequences of XDH have been determined in human [5, 13, 19, 20], cow [2], rat [1], mouse [15], chicken [14] and other species [3, 4, 7, 8, 12]. The amino acid sequences of $\mathrm{XDH}$ deduced from cDNA sequences suggested the conserved function among these species. However, there are no structural and functional informations of the XDHs in carnivorous animals. To extend our studies on purine metabolism in cat tissue, in the present study, we determined a cDNA sequence and characterized the deduced amino acid sequence in order to obtain structural information on feline $\mathrm{XDH}$.

Hepatic cells were obtained from a healthy cat by biopsy under anesthesia. A poly(A) RNA sample was prepared from the liver by acid guanidinium thiocyanate-phenolchloroform extraction(AGPC) methods and oligo(dT)-latex. Reverse transcription(RT) was carried out using oligo(dT) $)_{16}$ and AMV reverse transcriptase XL (Takara Shuzo co., Shiga, Japan) at $42^{\circ} \mathrm{C}$ for $60 \mathrm{~min}$. After reverse transcription, $\mathrm{XDH}$ cDNA including coding region was amplified by polymerase chain reaction(PCR). For PCR amplification, primers were prepared from the conserved sequences in human[Accession No. NM_000379], bovine [Accession
No. X98491 and X83508], rat [Accession No. J05579] and mouse [Accession No. X62932] XDHs presented in GenBank. PCR amplification was carried out using the primer sets of XDH-11(5'-TGGCAAAAAGGTGCTCGAGAAAAATGCAGA-3') and XDH-12(5'-GGAATGTCGACACAGTACACGGCCTC-3') for 5' coding region of $\mathrm{XDH}$ and $\mathrm{XDH}-21\left(5^{\prime}\right.$-AGCTTCTTCTTCAAGGTCGACCTGACAGT-3') and XDH-22(5'-CTGAGCTCGAGCTGCACGGATGGC-3') for 3' coding region. The expected length of PCR products using each primer set of 11-12 and 21-22 from human XDH cDNA was 1761 and 2316 bp, respectively. Primer XDH-14(5' -TTCTTCTCGAGTAGGCCAAAAGGG-3') and XDH-23(5'TGGCAGCCTCGAGCTTCTTCGCC-3') were used for 5', and 3' terminal regions by rapid amplified of cDNA end(RACE) method. For 5' RACE method, the addition of a number of dATP was carried out to the $5^{\prime}$ end of the first strand cDNA using terminal deoxynucleotidyl transferase (Life Technologies, Inc., U.S.A.). The PCR products were cloned in SalI site of pUC19 plasmid vector or in pT7BlueVector(Novagen, IW U.S.A.) and sequenced by employing Hitachi SQ5500 DNA sequencer (Nisseisangyo Co. Tokyo, Japan). All results were confirmed by two independent experiments for eliminating the point mutation due to the misincorporated base occurred in PCR amplification.

The clones from RT-PCR products covered 4931 bases of feline xanthine dehydrogenase mRNA [Accession No. AF286379]. An open reading frame of 4002 base pairs encoding 1333 amino acid residues from the initiation methionine to carboxyl-terminal valine was identified. The molecular weight and isoelectric point were calculated to be approximately $146 \mathrm{kDa}$ and 7.0 by DNASIS (Hitachi Software Engineering, Yokohama, Japan), which is similar to the size of XDHs from various mammalian species $[1,2,6$, 18]. A typical polyadenylation signal (AATAAA) was found in the large 3' untranslated region. The deduced 1333 amino acid residues of feline XDH shared approximately $90 \%, 87 \%, 87 \%$ and $86 \%$ identity with those of human, 
( A )

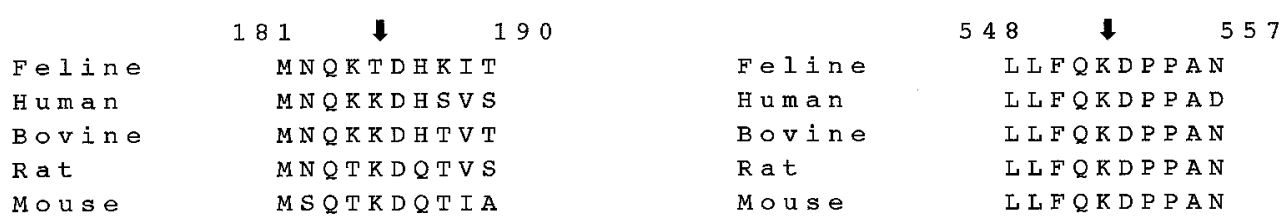

( B )

( C )

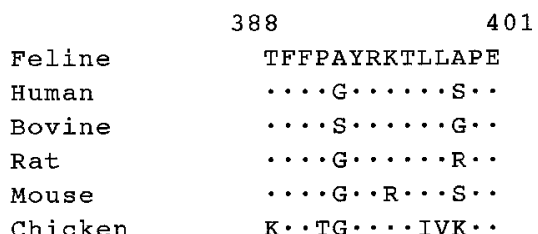

( $E)$

( D )

$\begin{array}{lcc} & 42 \quad 47 & 796 \quad 801 \\ \text { Feline } & \text { GCGEGG } & \text { GGGFG } \\ \text { Human } & \ldots \ldots & \ldots \ldots \\ \text { Bovine } & \ldots \ldots & \ldots \ldots \\ \text { Rat } & \ldots \ldots & \ldots \ldots \\ \text { Mouse } & \ldots \ldots & \ldots \ldots\end{array}$

Feline

Human

Bovine

Rat

Mouse
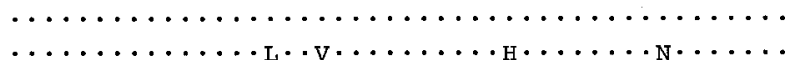

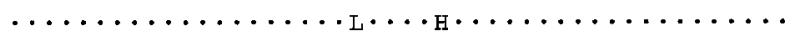

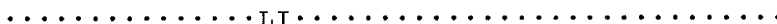

Fig. 1. Comparison of the deduced amino acid sequences from XDH cDNAs of various animals. Amino acid residues represent one-letter notation. The dots indicate amino acid residues identical to those of feline. The bars indicate gaps in the sequence alignment. The numbers above the feline amino acid sequences indicate the amino acid number from the initiation methionine deduced from feline XDH cDNA sequence. (A) Trypsin digestion sites in XDH. These regions were well conserved among mammalians. One point mutation is observed in feline $\mathrm{XDH}$, which suggests the change of digestion site in feline XDH. Arrows indicate digestion sites by trypsin. (B) N-terminal regions of $\mathrm{XDH}$ among feline, human, bovine, rat and mouse. Seven cysteine residues in the N-terminal regions that appear to contribute to the iron-sulfur centers are indicated by asterisks above the feline amino acid sequence. (C) NAD binding site regions determined in chicken liver XDH using 5'-p-fluorosulfonylbenzoyladenosine to mammalian corresponding sequences. (D) Two consensus amino acid sequences of GXGXXG, which contribute the conformation for NAD bindings site. (E) Proposed molybdenum binding sites in XDH among animal species. 
bovine, rat and mouse XDH, respectively.

The XDH from rat liver and bovine milk was cleaved into three peptides by trypsin digestion $[1,2]$. An N-ternimal 20 $\mathrm{kDa}$ domain contained two iron-sulfur centers. A C-terminal $85 \mathrm{kDa}$ domain was considered to bind the pterin molybdenum cofactor. The NAD binding site was estimated to be existed in a central $40 \mathrm{kDa}$ domain [10]. The trypsin cleaved after two lysine (Lys) residues (Lys-184 and Lys539 ) in rat XDH [1]. The purified rat XDH of NAD-dependent type enzyme was irreversibly converted to the $\mathrm{O}_{2}$ dependent type by digestion with trypsin. The two corresponding Lys residues were perfectly conserved in human, bovine, rat and mouse. Although the corresponding Lys539 (Lys-552 in feline XDH) was detected in feline XDH, the corresponding 185 th residue was threonine (Thr) instead of Lys (Fig. 1A). The Lys residue is considered to be attacked by trypsin digestion and be responsible for dehydrogenase to oxidase conversion. The amino acid substitution in the corresponding position in the feline XDH might induce the change of digestion site by proteases and suggest the functional differences related to the conversion from $\mathrm{NAD}$-dependent type to the $\mathrm{O}_{2}$-dependent type. The $\mathrm{N}$-terminal regions of human, bovine, rat and mouse XDHs are appeared to contain two iron-sulfur centers, which are formed through the contribution of cysteine (Cys) residues. The rich Cys residues were also found in the N-terminal region in feline $\mathrm{XDH}$. The comparison of the N-terminal regions in $\mathrm{XDH}$ amino acid sequences between feline and other mammalian species indicated the remarkable high homology (Fig. 1B). The matching percentage of the deduced amino acid of feline XDH N-terminal region was estimated to be $94 \%, 92 \%, 90 \%$ and $88 \%$ to those of human, bovine, rat and mouse, respectively. The N-terminal regions of feline $\mathrm{XDH}$ is also considered to play a role for iron-sulfur. $\mathrm{XDH}$ have one binding site of the nucleotide cofactor $[11,18]$. The putative NAD binding site was also conserved in feline XDH at residues 388-401 (Fig. 1C). Two consensus amino acid sequences of Gly-Xaa-Gly-XaaXaa-Gly have been proposed to provide the conformation essential for NAD binding [17]. The consensus sequences contribute the $\beta \alpha \beta$ fold formation and an NAD binding site is formed around the sequence. The corresponding amino acid sequence is also found in the deduced sequence from feline XDH cDNA at 42-47 and 796-801 residues. This consensus sequence was perfectly conserved in amino acid sequences in feline, human, bovine and rat and mouse XDHs, which suggested the functional contribution (Fig. 1D). The possible candidate for the molybdenum binding site was proposed to be located in the C-terminal of XDH and other molybdo-enzymes [18]. The high homologous sequence was also identified in the C-terminal region of a feline XDH cDNA at amino acid residues 793-838 (Fig. 1E). Comparison of the deduced molybdenum binding site among mammalian species indicated that the feline corresponding sequence was $100 \%, 91 \%, 95 \%$ and $95 \%$ identical to those of human, bovine, rat and mouse XDH cDNA, respectively.
In conclusion, the comparison of XDH cDNA sequences and the deduced amino acid residues demonstrated high homology among mammalian species and the well conservation of the functional sequences for two iron-surfur centers, one NAD binding site and one molybdenum center. However, the amino acid substitution of a Lys was demonstrated in the well-conserved sequence of a feline $\mathrm{XDH}$ cDNA, which suggested the possiblity of the change in trypsin digestion related to the conversion from NADdependent type to $\mathrm{O}_{2}$-dependent type. Feline XDH might be different from other mammalian XDHs in the conversion system and the functional role. The determination of feline $\mathrm{XDH}$ cDNA sequence should be useful in future studies on the feline purine metabolism, the disease with XDH abnormality and the role of $\mathrm{XDH}$ in pathological condition as ischemia-reperfusion injury in various organs.

\section{REFERENCES}

1. Amaya, Y., Yamazaki, K., Sato, M., Noda, K., Nishino, T. and Nishino, T. 1990. J. Biol. Chem. 265: 14170-14175.

2. Berglund, L., Rasmussen, J.T., Andersen, M.D., Rasmussen, M.S. and Petersen, T.E. 1996. J. Dairy Sci. 79: 198-204.

3. Glatigny, A. and Scazzocchio, C. 1995. J. Biol. Chem. 270: 3534-3550.

4. Houde, M., Tiveron, M.C. and Bregegere, F. 1989. Gene 85: $391-402$.

5. Ichida, K., Amaya, Y., Noda, K., Minoshima, S., Hosoya, T., Sakai, O., Shimizu, N. and Nishino, T. 1993. Gene 133: 279284.

6. Ikegami, T. and Nishino, T. 1986. Arch. Biochem. Biophys. 247: 254-260.

7. Keith, T.P., Riley, M.A., Kreitman, M., Lewontin, R.C., Curtis, D. and Chambers, G. 1987. Genetics 116: 67-73.

8. Lee, C.S., Curtis, D., McCarron, M., Love, C., Gray, M., Bender, W. and Chovnick, A. 1987. Genetics 116: 55-66.

9. McCord, J.M. 1985. New Engl. J. Med. 312: 159-163.

10. Nishino, T. and Nishino, T. 1989. J. Biol. Chem. 264: 54685473.

11. Nishino, T. 1994. J. Biochem. (Tokyo) 116: 1-6.

12. Riley, M.A. 1989. Mol. Biol. Evol. 6: 33-52.

13. Saksela, M. and Raivio, K.O. 1996. Biochem. J. 315(Pt 1): 235-239.

14. Sato, A., Nishino, T., Noda, K., Amaya, Y. and Nishino, T. 1995. J. Biol. Chem. 270: 2818-2826.

15. Terao, M., Cazzaniga, G., Ghezzi, P., Bianchi, M., Falciani, F., Perani, P. and Garattini, E. 1992. Biochem. J. 283(Pt 3): 863870.

16. Waud, W.R. and Rajagopalan, K.V. 1976. Arch. Biochem. Biophys. 172: 365-379.

17. Wierenga, R.K., De Maeyer, M.C.H. and Hol, W.G.J. 1985. Biochemistry 24: 1346-1357.

18. Wootton, J.C., Nicolson, R.E., Cock, J.M., Walters, D.E., Burke, J.F., Doyle, W.A. and Bray, R.C. 1991. Biochim. Biophys. Acta. 1057: 157-185.

19. Wright, R.M., Vaitaitis, G.M., Wilson, C.M., Repine, T.B., Terada, L.S. and Repine, J.E. 1993. Proc. Natl. Acad. Sci. U. S. A. 90: 10690-10694.

20. Xu, P., Huecksteadt, T.P., Harrison, R. and Hoidal, J.R. 1994. Biochem. Biophys. Res. Commun. 199: 998-1004. 